EDITORIAL

\title{
The changing face of infective endocarditis
}

\author{
E Cecchi, M Imazio, R Trinchero
}

Heart 2006;92:1365-1366. doi: 10.1136/hrt.2006.092635

Infective endocarditis is a disease that continues to evolve in response to changing host conditions and other factors

nfective endocarditis (IE) is a relatively rare disease that, as already reported by William Osler in $1885,{ }^{1}$ may develop in different ways and have several clinical presentations, yet always comprises the same four fundamental physiopathological elements: persistent bacteraemia with an appropriate infectious microorganism, predisposing factors, active endomyocardial involvement, and vascular phenomena.

In the last 30 years noteworthy progress has been achieved in our understanding of IE through epidemiologic studies, use of clinical and instrumental diagnostic criteria for a more accurate case definition, and the use of echocardiography for the diagnosis and management of the disease. Despite medical preventive strategies, and advances in technology and medical and surgical treatment, the incidence of IE is almost unchanged and is still associated with high morbidity and mortality rates. ${ }^{2-5}$

During the last 50 years the condition's aetiology has undergone a major change as a consequence of important social transformations in western countries, such as aging and the increasing role of degenerative valve disease, the virtual disappearance of rheumatic heart disease, and the increasing role of co-morbidities, longer hospital stays, and more invasive therapies which are responsible for an increasing number of sepsis cases with nosocomial-nosohussial IE. ${ }^{2}$

Above all, in urban areas but with wide regional variations, intravenous drug use has become responsible for sepsis and IE, with unusual features. Cutaneous, endovascular, genitourinary, and intestinal routes have become increasingly important beside the traditional oral route. This change is reflected by the decrease of oral streptococci together with the increase of Staphylococcus aureus, coagulase-negative staphylococci, enterococci, and Streptococcus bovis infections. ${ }^{2} 6-13$ Insidious changes in the infectious microorganisms have given rise to the development of global antibiotic resistance. ${ }^{2}{ }^{3}$

Echocardiography has enabled improved study of the endomyocardial damage, increasing the diagnostic capability. Not surprisingly observational studies report a changing epidemiological and clinical picture of IE, though important regional differences have been reported, reflecting heterogeneous populations. It is now well known that IE is more frequent in older populations and in urban industrialised areas, where drug addiction and increasing use of medical procedures play an important role in the development of the disease. ${ }^{2} 81112$ Limited data are available on other subgroups at risk of IE, such as those with congenital heart disease, where the frequency of endocarditis could be increased by longer survival and more complex surgical therapy with the use of prosthetic material. ${ }^{13}$

In this issue of Heart, two papers present different evolutionary processes of the clinical epidemiology of IE in varied populations: the first one in the general adult population, ${ }^{14}$ and the second one in a subgroup with congenital heart disease. ${ }^{15}$

\section{FINNISH STUDY}

The study by Heiro et $a l^{14}$ is a retrospective observational study on 326 episodes of IE treated during 1980-2004 in Turku, in the south-west of Finland. The teaching hospital is a tertiary referral centre with a cardiothoracic surgical department, and provides primary care for infectious diseases for about 200000 inhabitants. The study is a rigorous observation with a wide analysis of clinical variables and short-term prognosis, and adequate statistical analysis from a well-motivated group. Contrary to the majority of recent observations, this study shows few epidemiological and clinical changes over 25 years: aging of patients with IE, drug addiction in $7 \%$ of cases after 1994, $S$ areus surpassing Streptococcus viridans after 1995 due to intravenous drug addiction, and some cases associated with haemodialysis. Surprisingly there is no trend toward an increase in prosthetic IE. The authors do not report an increase in coagulase-negative staphylococci, enterococci, or $S$ bovis.

The first consideration is that the studied population behaves as a general population, despite cases from the referral cardiothoracic centre. Thus, the incidence of IE is 13.6 cases/ year and is slightly more than expected for 200000 inhabitants, where not all possible IE cases included in the study according to Duke criteria are true IE cases.

The only similar experience has been recently reported in Minnesota with even more striking data. ${ }^{16}$ In this well conducted study in a rural area involving white subjects with a low percentage of drug addicts, only marginal changes of the disease have been reported over 30 years of observation. These two experiences should be considered carefully but cannot be generalised upon. Other studies include data before 1995 and do not report the important changes over the last decade.

\section{NOSOCOMIAL ENDOCARDITIS}

Unfortunately the retrospective nature of the study by Heiro et $a l^{14}$ does not provide definitive 
data on nosocomial, nosohussial IE that, despite important regional variations, ${ }^{11} 12$ is described as increasing and posing new challenges in Western countries. In fact the authors suggest a possible marginal role for nosocomial endocarditis, because the increase of $S$ aureus cases is only due to drug addiction and haemodialysis. It is possible that the lack of epidemiological changes may be explained by the relative lack of several nosocomial cases. These data may be an expression of a regional variation, as in Minnesota, which is probably connected to socioeconomic and health peculiarities of those populations. Undoubtedly those are populations with a good socioeconomic standard, and low frequency of drug addiction. In Finland, HIV infection is rare outside Helsinki, and several health preventive initiatives have been proposed. If these results are confirmed in prospective series, it will be necessary to study carefully why these areas are preserved from dangerous nosocomial and nosohussial IE. It is possible that these findings may be an expression of less invasive treatments and more preventive aids. On the other hand it is well known that in some areas, invasive vascular procedures are sometimes widespread well beyond established indications. Guidelines should consider these new epidemiologic aspects.

Moreover, generalisations cannot be made about the data from Heiro et al, ${ }^{14}$ as demonstrated by several published studies where important clinical and epidemiological changes in IE were reported over the last decades, ${ }^{2-12}{ }^{14}$ despite being from different backgrounds and centres. This constant evolution represents one of the most intriguing challenges in this field.

\section{CONGENITAL HEART DISEASE}

The second paper by Di Filippo et al ${ }^{15}$ points out the epidemiological and clinical changes of IE in patients with congenital heart disease. These patients are a small but definite subgroup of patients within available surveys of IE, that deserve an in-depth analysis because of their unusual ages (children or young adults) and wide diversity. This article is a retrospective study on 153 episodes of IE from 1966 to 2001 that shows an increase in the mean age at onset, and a trend toward a higher frequency of endocarditis in patients with small, not operated on double-inlet ventricles (DIV), complex cyanotic diseases with palliative therapies, and Rastelli operation. The main route remains the oral one, but with an increase of cases related to cutaneous routes. The main isolated microorganisms are streptococci, and survival does not change over time in a significant way. Prophylaxis was seldom performed, and sometimes had been ineffective.

This study confirms that the host is mainly responsible for these changes. Surgical therapy is a contributing factor, increasing the survival rate, the aging of patients, and the risk of endocarditis after operations with prosthetic material or residual high velocity shunts. On the other hand, surgery may reduce the risk of endocarditis in a wide range of operated patients. Detailed data are not available on nosocomial endocarditis, but it seems uncommon in those young patients where streptococci and the oral route are prevalent, and common in patients with known heart disease.

Both studies show that IE is a disease in constant evolution, with unusual features according to regional variations or subgroups. Certainly these results cannot be generalised, but may show the existence of subgroups with a low rate of drug addiction, high prevalence of the oral route, and streptococci. Study of regional differences and population subgroups, including referral and non-referral centres, may offer an explanation for some of these changes and propose possible solutions for the future.

\section{Authors' affiliations}

E Cecchi, M Imazio, R Trinchero, Cardiology Department, Maria Vittoria Hospital, Torino, Italy

No financial disclosure

No conflict of interest

\section{REFERENCES}

1 Osler W. Gulstonian lectures on malignant endocarditis. Lecture I. Lancet 1885;1:415-8

2 Cabell $\mathrm{CH}$, Abrutyn E. Progress toward a global understanding of infective endocarditis. Lessons from the International Collaboration on Endocarditis. Cardiol Clin 2003;21:147-58.

3 Moreillon P, Que Y-A. Infective endocarditis. Lancet 2004;363:149-59.

4 Habib G. Management of infective endocarditis. Heart 2006;92:124-30.

5 Vikram H, Buenconsejo J, Hasbun R, et al. Impact of valve surgery on 6-month mortality in adults with complicated, left sided, native valve endocarditis. JAMA 2003;290:3207-14.

6 Hoen B, Alla F, Selton-Suty C, et al. Changing profile of infective endocarditis. Results of a 1-year survey in France. JAMA 2002;288:75-81.

7 Hogevik H, Olaison L, Anderson R, et al. Epidemiologic aspects of infective endocarditis in an urban population: a 5 year prospective study. Medicine (Baltimore) 1995;74:324-39.

8 Fowler WG, Miro JM, Hoen B, et al for the ICE Investigators. Staphylococcus aureus endocarditis. A consequence of medical progress. JAMA 2005;293:312-21.

9 Cecchi E, Forno D, Imazio $M$, et al. The changing epidemiology and clinical features of infective endocarditis in Piemonte. It Heart J 2004;5:249-56.

10 Martin-Davila P, Fortun J, Navas E, et al. Nosocomial endocarditis in a tertiary hospital: an increasing trend in native valve cases. Chest 2005; 128:772-9.

11 Martin GS, Mannino DM, Eaton S, et al. The epidemiology of sepsis in the United States from 1979 through 2000. New Engl J Med 2003;348:1546-54.

12 Horstkotte D, Follath F, Gutschik E, et al. Task Force Members on Infective Endocarditis of the European Society of Cardiology; ESC Committee for Practice Guidelines (CPG); Document Reviewers. Guidelines on prevention, diagnosis and treatment of infective endocarditis executive summary; the task force on infective endocarditis of the European Society of Cardiology. Eur Heart J 2004;25:267-76.

13 Naiwa K, Nakazawa M, Tateno S, et al. Infective endocarditis in congenital heart disease: Japanese national collaboration study. Heart 2005;91:795-800.

14 Heiro $M$, Helenius $H$, Mäkilä S, et al. Infective endocarditis in a Finnish teaching hospital: a study on 326 episodes treated during 1980-2004. Heart 2006;92:1457-62.

15 Di Filippo S, Delahaye F, Semiond B, et al. Current patterns of infective endocarditis in congenital heart disease. Heart 2006;92:1490-5.

16 Tleyjeh IM, Steckelberg JM, Murad HS, et al. Temporal trends in infective endocarditis: a population-based study I Olmsted County, Minnesota. JAMA 2005;293:3022-8 